\title{
Immunoendocrine Interactions during HIV-TB Coinfection: Implications for the Design of New Adjuvant Therapies
}

\author{
Guadalupe Veronica Suarez, ${ }^{1}$ Maria Belen Vecchione, ${ }^{1,2}$ \\ Matias Tomas Angerami, ${ }^{1}$ Omar Sued, ${ }^{3,4}$ Andrea Claudia Bruttomesso, ${ }^{2}$ \\ Oscar Adelmo Bottasso, ${ }^{5}$ and Maria Florencia Quiroga ${ }^{1}$ \\ ${ }^{1}$ Instituto de Investigaciones Biomédicas en Retrovirus y SIDA, University of Buenos Aires School of Medicine, \\ C1121ABG Buenos Aires, Argentina \\ ${ }^{2}$ Unidad de Microanálisis y Métodos Físicos Aplicados a Química Orgánica (UMYMFOR), Department of Organic Chemistry, \\ University of Buenos Aires School of Sciences, C1428EGA Buenos Aires, Argentina \\ ${ }^{3} J . A$. Fernández Hospital, Infectious Diseases Unit, C1425AGP Buenos Aires, Argentina \\ ${ }^{4}$ Huesped Foundation, C1202ABB Buenos Aires, Argentina \\ ${ }^{5}$ Instituto de Inmunología Clínica y Experimental de Rosario (IDICER), CONICET-UNR, Rosario, S2002LRL Santa Fe, Argentina
}

Correspondence should be addressed to Maria Florencia Quiroga; florenciaquiroga@gmail.com

Received 19 December 2014; Revised 30 April 2015; Accepted 1 May 2015

Academic Editor: Kuldeep Dhama

Copyright (C) 2015 Guadalupe Veronica Suarez et al. This is an open access article distributed under the Creative Commons Attribution License, which permits unrestricted use, distribution, and reproduction in any medium, provided the original work is properly cited.

\begin{abstract}
Worldwide, around 14 million individuals are coinfected with both tuberculosis (TB) and human immunodeficiency virus (HIV). In coinfected individuals, both pathogens weaken immunological system synergistically through mechanisms that are not fully understood. During both HIV and TB infections, there is a chronic state of inflammation associated to dramatic changes in immune cytokine and endocrine hormone levels. Despite this, the relevance of immunoendocrine interaction on both the orchestration of an effective immune response against both pathogens and the control of the chronic inflammation induced during HIV, TB, or both infections is still controversial. The present study reviews immunoendocrine interactions occurring during HIV and TB infections. We also expose our own findings on immunoendocrine cross talk in HIV-TB coinfection. Finally, we evaluate the use of adrenal hormones and their derivatives in immune-therapy and discuss the use of some of these compounds like the adjuvant for the prevention and treatment of TB in HIV patients.
\end{abstract}

\section{Introduction}

Worldwide, around 14 million individuals are believed to be coinfected with both tuberculosis (TB) and human immunodeficiency virus-1 (HIV-1), constituting together the leading infectious diseases in resource-limited countries [1].

The African region accounted for most of the HIVinfected TB cases, and TB is the first cause of death in AIDS context $[1,2]$. Also, HIV coinfection raises latent TB reactivation risk by 20 -fold, becoming the leading risk factor for Mycobacterium tuberculosis (Mtb) infection progression to active disease $[1,3]$. Likewise, TB exacerbates HIV infection progression $[4,5]$. Therefore, the intersecting HIV and tuberculosis epidemics in countries with a high disease burden of both infections pose many challenges and opportunities.

In coinfected individuals, $M t b$ and HIV, weaken immunological system synergistically, disrupting immune functions through mechanisms that are not fully understood.

An extended TB treatment ( 6 months) is required to eradicate $M t b$ infection, rising up to 9 months for HIV patients [6]. While replicating bacilli are killed by anti-TB drugs during the first weeks of treatment, $M t b$ evades drugs and host-immune responses by successfully adapting to a quiescent physiological state [7]. In the absence of chemotherapy 
treatment, immune system failure, among other factors, leads to TB relapse [8]. Therefore, a more effective and shorter treatment may require the modulation of the immune response towards a protective phenotype. Actually, several immune modulation strategies, like vitamin D supplement [9], treatment with Th1 cytokines $[10,11]$, and immune suppression to disrupt granulomas and make bacteria more susceptible to chemotherapy [12], among others, have been explored with diverse results [13].

Endocrine and immune systems are strictly connected by multiple mutual regulatory pathways, the most extensively studied of which is the anti-inflammatory and immune-suppressive action of glucocorticoids (GCs). Several cytokines have been found to regulate the endocrine system and many steroid and nonsteroid hormones have shown immunomodulatory effects [14-17].

In a broad range of bacterial, viral, and parasitic infections, the immune response developed against the pathogen is paralleled by a significantly altered hormonal response both in experimental models and human patients [18]. In fact, there is some evidence that supports a role for the interaction between immune and endocrine systems in the orchestration of an effective defense against the infectious agents $[18,19]$. Despite this, the relevance of the cross talk between immune and endocrine systems on the defense against infections has not been fully elucidated.

The classical view stands that proinflammatory cytokines (mostly IL-1 $\beta$, IL-6, TNF- $\alpha$, and IFN- $\alpha / \beta$ ) produced in response to pathogens induce the hypothalamic-pituitaryadrenal (HPA) axis for the release of GCs $[14,18]$. In turn, GCs suppress the immune system at several levels, avoiding the possible adverse effects of an exacerbated immune response and helping to terminate it once the injurious agent was cleared [20]. However, although true for some particular situations like acute inflammation, the view of GCs such as simple immune suppressors has been changed, as there is growing evidence showing that GCs may be both pro- and antiinflammatory, and also even necessary for several immune processes [20].

On the other hand, the activation of HPA axis induces the release of other adrenal hormones, like dehydroepiandrosterone (DHEA) and DHEA-sulphate (DHEA-s), with known anti-GC functions [21]. GCs and DHEA have opposite effects on adaptive immune cells. While GCs inhibit both Th1 and Th2 cytokines by activated human T cells, its effect on Th1 cytokines is more pronounced, shifting $\mathrm{T}$ cell response towards Th2 profiles [22]. On the contrary, DHEA promotes a shift towards Th1 responses by skewing cytokine production, upregulates Th1 cytokines like IL- 2 and IFN- $\gamma$, and downregulates Th2 cytokines like IL-4 and IL-5 [23]. In line with this, DHEA and its derivatives have been proved to enhance protective immune responses against several pathogens [24-27].

During infection, immune-endocrine interactions are even more complex, since pathogens per se can modulate endocrine function either by releasing soluble factors or by directly colonizing endocrine tissue $[28,29]$. Furthermore, specific treatments like antibiotics may affect hormone metabolism [30]. Moreover, during chronic infections like HIV or $\mathrm{TB}$, in which pathogens are not cleared and immune response must be sustained in time, there is a chronic state of inflammation associated to dramatic changes in immune cytokine and endocrine hormone levels [29, 31]. Despite this, the relevance of immunoendocrine interaction on both the orchestration of an effective immune response against both pathogens and the control of the chronic inflammation is still controversial.

The present study reviews immunoendocrine interactions occurring during HIV and TB infections. In addition, we expose our own findings on immunoendocrine cross talk in HIV-TB coinfection. Finally, we evaluate the use of adrenal hormones and their derivatives in immune-therapy and discuss the use of some of these compounds like the adjuvant for the prevention and treatment of TB in HIV patients.

\section{Immunoendocrine Alterations during Human Tuberculosis}

Initial infection with $M t b$ occurs upon inhalation of airborne droplets containing bacilli, which, once in the lungs, infect and reside mainly in myeloid cells, especially alveolar macrophages [7]. In most people, such initial infection resolves, or it is kept under control by the development of an appropriate adaptive immune response that prevents bacillary proliferation and dissemination.

Effective control of $M t b$ infection relies on cell-mediated immunity [32]. Once in the infected tissue, $\mathrm{T}$ cells produce interferon gamma (IFN- $\gamma$ ) in response to mycobacterial antigen presentation by antigen presenting cells and consequently IFN- $\gamma$ activates macrophages to kill intracellular bacteria $[33,34]$. In addition to CD4+ T cells, which are thought to be the most important, both CD8+ T cells $[35,36]$ and CD1-restricted unconventional T cells [37] contribute to the effective control of $M t b$ infection. Both CD8+ T cells and CD1-restricted unconventional T cells are thought to be particularly important in the prevention of latent $\mathrm{TB}$ reactivation [35-38].

Moreover, at least $30 \%$ of the persons that take contact with $M t b$ remain persistently infected without exhibiting signs of disease in a state known as latent TB infection (LTBi) [39]. While infection may persist for life, bacillary growth may be reactivated in $5-10 \%$ of non HIV-infected subjects, probably due to factors affecting the host's immune status, and postprimary disease ensues [7]. As described above, in HIV-infected persons this rate rises to $20 \%$. Persistent infection with $M t b$ is accompanied by chronic, low-level inflammation, which together drive to several changes in cytokine and hormone levels.

TB patients show a twofold increase in IFN- $\gamma$ and IL-10 and a 10-fold increase in IL-6 plasma levels, accompanied by a $50 \%$ decrease in testosterone and DHEA in plasma when compared to healthy controls [40]. Also, TB patients show higher diurnal peaks of cortisol [41] and higher cortisol responses to ACTH stimulation [42], showing that HPA axis is activated in these patients. Since GCs enhance Th2 activity, HPA axis control may be relevant to the Th1/Th2 balance during the establishment of the anti-infectious immune response $[33,34,43]$. Conversely, DHEA can directly and indirectly 
enhance Th1 T cell activity [21]. According to this, Mtbspecific IFN- $\gamma$ secretion in TB patients correlated positively with DHEA plasma levels and inversely with cortisol/DHEA ratio [44].

In addition to proinflammatory cytokines, regulatory cytokines such as TGF- $\beta$ are likely involved in the control of some endocrine functions during TB infection. For instance, in vitro treatment of the human adrenal cell line NCI-H295-R with culture supernatants of $M t b$-stimulated PBMC from TB patients inhibited DHEA and induced cortisol secretion [40]. Of note, treatment with anti-TGF- $\beta$ antibodies abolished the inhibitory effect on DHEA but not cortisol secretion [45].

Overall, the hormonal changes occurring during $M t b$ infection could prevent the development of protective immune responses and the control of persistent inflammation.

\section{Immunoendocrine Interactions in HIV Infection}

HIV-1 infection is accompanied by a strong innate and adaptive immune response, beside which it remains as a chronic infection through lifetime.

The main obstacle for HIV eradication is the establishment of a latent HIV reservoir, which occurs during the very early stages of primary infection. Virus genomic integration and latency, as well as an enormous genetic diversity, propel a constant immune escape which limits HIV-specific immune response efficacy, especially during the early events of infection.

HIV infection causes the depletion of CD4+ T cells, which accounts for most infection hallmark symptoms. Additionally, a systemic and chronic immune activation and an accelerated $\mathrm{T}$ cell turnover are also thought to contribute to HIV disease progression [46]. Finally, the constant antigenic stimulation is thought to induce $\mathrm{T}$ cell dysfunction in response to antigen stimulation [47].

Despite the persistent immune escape seen in the great majority of HIV-infected people, there are rare individuals who control HIV-1 burden to undetectable levels. These individuals, known as elite controllers, show HIV-specific CD8+ $\mathrm{T}$ cells with higher cytotoxic capacity $[48,49]$ and are also associated to some specific MHC class I alleles [50]. In line with this, HIV infection progression was suggested to be associated with a reduction in cellular immunity, that is, Th1, and an increase in Th2 cytokine production $[51,52]$.

Considering that GCs provoke a shift from Th1 to Th2 immunity and also alter the balance between Th17 and Treg cells [53], recently implicated in the pathogenesis of HIV [54], HPA axis activation could play a central role in the progression of HIV infection to disease. In fact, increased basal ACTH and/or cortisol levels in 50\% of HIV-infected patients accompanied by an impaired ACTH and cortisol response to stress and $\mathrm{CRH}$ challenge, especially in advanced disease, have been reported $[55,56]$. The proposed mechanisms to this hypercortisolemia include a shift in steroidogenesis from DHEA and aldosterone to cortisol and the stimulation of hypothalamus, pituitary, and adrenal cortex by cytokines [ 56 , 57]. Moreover, the stimulation of the HPA axis may also result from the stimulatory effects of viral proteins, like the HIV envelope protein gp-120 and the structural protein viral protein $\mathrm{R}$ (Vpr) which have been shown to increase serum ACTH and to cause GCs hypersensitivity, respectively [58-60].

HIV-infected patients show a characteristic pattern of high cortisol and low DHEA-s levels [61], which have been associated with a negative course of disease $[55,61]$. In fact, it has been shown that DHEA-s levels decline with the progression of the disease in parallel with the CD4 T cell count [61]. Immune system deterioration in HIV-infected patients with high cortisol/DHEA-s levels seems to be associated with the suppression of Th1 cytokines and the concomitant increase in Th2 cytokines levels [62]. In line with this, it has been suggested that cortisol-resistant HIV-infected patients have a prominent Th1 cytokine profile and, therefore, present a limited progression to AIDS $[63,64]$.

\section{Immunoendocrine Alterations Observed in HIV-TB Coinfection}

There is limited data available about the role of adrenal steroids on the immune response developed by patients coinfected with HIV-1 and Mtb. We determined DHEA, DHEA-s, and cortisol plasma levels and the role of these adrenal hormones on $M t b$-specific Thl responses and Treg frequencies in patients dually infected with $M t b$ and HIV undergoing different stages of $M t b$ infection [65]. We observed that, in HIV-infected patients with active TB (HIV-TB), DHEA plasma levels were diminished by twofold compared to HIVinfected patients without $M t b$ coinfection or healthy donors. On the contrary, HIV-infected patients latently infected with $M t b$ (HIV-LTBi) showed preserved DHEA levels. Additionally, while cortisol plasma levels were slightly higher in HIVTB patients than in HIV patients, cortisol/DHEA ratio was almost 4 times higher in HIV-TB patients compared to HIV, HIV-LTBi, and healthy donors. Of note, in HIV-TB patients, DHEA-s levels correlated positively, while cortisol plasma levels correlated negatively with CD4+ T cell count. Moreover, we observed an inverse correlation between DHEA-s plasma levels and Treg frequency in the same group.

A remarkable finding of this study was the persistent observation of a conspicuous CD4+CD25-FoxP3+ population in HIV-TB patients that was not observed in the other groups. Notably, CD4+CD25-FoxP3+ population is also increased in systemic lupus erythematosus patients [66], but its nature remains undetermined. Some authors suggested that these cells are FoxP3+ non-Treg T cells [67], whereas others argue that these cells are dysfunctional Treg cells with limited regulatory potential [68].

In order to clarify this, we evaluated FoxP3 liability in these cells by keeping them unstimulated for different periods of time. By doing this, we observed that within these cells Foxp3 expression was relatively stable, suggesting that, at least in HIV-TB patients, the intriguing CD4+CD25-FoxP3+ population is not a transiently activated effector population and rather might have regulatory functions $[67,68]$. We hypothesize that this unusual regulatory population may preclude protective immune responses against $M t b$ in our cohort 
of coinfected patients, a topic currently under investigation by our group. Finally, we also found that the frequency of CD4+CD25-FoxP3+ cells in HIV-TB patients negatively correlated with DHEA plasma levels, which is consistent with a role of DHEA on enhancing Th1 responses while diminishing this particular regulatory $\mathrm{T}$ cell population.

Moreover, we also found that the initiation of antituberculous treatment (ATT) diminished CD4+CD25-FoxP3+, which was restored to normal levels after finalization of treatment. In contrast, conventional CD4+CD25+FoxP3+ Tregs in HIV-TB patients tended to diminish across visits, but not significantly. In HIV-TB patients, adrenal hormone balance was not restored as well, at least after 6 months of ATT (unpublished results).

In some HIV-infected patients, the recovery of specific immune responses during the beginning of highly active antiretroviral treatment (HAART) can elicit systemic inflammatory responses which lead to the establishment of the immune reconstitution inflammatory syndrome (IRIS). In HIV-TB IRIS patients, DHEA-s plasma levels were three times lower and cortisol/DHEA ratio was up to four times higher compared to the non-TB groups. The CD4+CD25FoxP3+ frequency was also increased in IRIS patients and negatively correlated with DHEA-s plasma levels [65].

Previous results obtained by our research group also showed a positive correlation between DHEA plasma levels and the frequency of a terminally differentiated population of CD8+ T cells in HIV-TB patients, which is thought to be crucial in preventing TB reactivation (in press and [38]). We also observed that in vitro DHEA treatment increased $M t b$ specific CD8+ T cell proportions and terminal differentiation in CD8+ T cells of HIV-TB coinfected patients. Additionally, we found that DHEA in vitro increased the expression of the transcription factor Tbet and Tbet/Eomesodermin ratio in isolated CD8+ cells, both known to drive to terminal differentiation in CD8+ T cells [69].

Based on our results, in HIV-TB coinfected patients, HPA axis displays a noticeable alteration, probably influenced by the chronic state of inflammation induced by both pathogens concomitantly. This condition may contribute to the immune system dysfunction seen in these patients.

\section{Adrenal Steroids in Immunotherapy}

As suggested by the evidence depicted above, DHEA seems to have two different functions regarding immune modulation: it favors Th1 responses, fundamental in the protection against intracellular pathogens like $M t b$ and HIV, and, on the other hand, it exerts anti-inflammatory actions, which may be beneficial in the context of chronic infection. For that reason, DHEA has been proved as an adjuvant therapy for the treatment of several infectious diseases.

The apparent dual function of DHEA, together with the fact that a cell receptor is not known for it, suggests that this hormone may exert its immune regulatory function through some of its metabolites, which are known to be potent immune-regulators in vivo [26]. DHEA is the physiological precursor for the synthesis of androgens and estrogen, but it can also be metabolized into oxygenated derivatives in nonsteroidogenic tissues, especially in human liver [70]. There is also evidence that DHEA can be converted into oxygenated derivatives like $3 \beta, 17 \beta$-androstenediol (AED) and $3 \beta, 16 \beta, 17 \beta$-androstenetriol (AET) by monocyte-derived macrophages [71].

In mice, DHEA and AED were protective against lethal dose of Pseudomonas aeruginosa or Enterococcus faecalis infection. Both steroids appeared to act on immune system rather than bacteria directly, since the growth of bacterium was not altered in in vitro assays [72]. DHEA, AET, and AED also proved to protect mice against lethal doses of human herpes type 2 or coxsackievirus B4 [26, 72] while DHEA supplementation enhanced the immune response against Trypanosoma cruzi in mice, as shown by a significant reduction in parasitemia levels [73].

A synthetic derivate from DHEA, $16 \alpha$-Bromoepiandrosterone (HE2000), has been tested in animal models of TB [25]. In these sets of experiments, HE2000 proved to reduce bacterial load associated with progressive TB and lowered IL- 4 expression associated with $M t b$ infection. Also, BALB/c mice with active TB treated with HE2000 showed lower bacterial proliferation and a higher Th1/Th2 cytokine balance. The treatment with HE2000 also resulted in lower percentages of lungs involved in pneumonia and higher bacterial clearance, when administered as an adjunct to conventional chemotherapy [25].

The above mentioned evidence suggests that DHEA and its derivate upregulate host immunity, leading to higher resistance to infectious agents. In line with this, treatment of mice with AET augmented the absolute numbers of CD4+/CD8+ $\mathrm{T}$ cells after irradiation and increased IL-2, IL-3, and IFN$\gamma$ levels counteracting hydrocortisone immune suppression [26]. Additionally, zinc and DHEA supplementation augmented macrophage count and IFN- $\gamma$ and NO concentrations in an additive manner [24].

Despite the above mentioned immune-enhancing effects of DHEA, the effect of this steroid on T cell function is controversial. While some researchers showed that DHEA enhanced $\mathrm{T}$ cells proliferation and IL-2 production [70], others reported the contrary [74]. A posterior study suggested that DHEA acted differentially on T and B lymphocytes, since it significantly inhibited proliferation of $\mathrm{T}$ cells, but, on the other hand, increased $\mathrm{B}$ cell mitogen pokeweed mitogen (PWM) effect on PBMC [75].

Experiments performed by our group demonstrated that DHEA induced the increment in the production of IL12 and reduced IL-10 secretion by $M t b$-stimulated human monocyte-derived dendritic cells (DCs) promoting Th1 responses [76]. Also, DHEA enhanced the expression of MHC I, MHC II, and CD86 and increased ERK1/2 phosphorylation. Furthermore, DHEA enhanced the antigen-specific $\mathrm{T}$ cell proliferation and IFN- $\gamma$ production induced by $M t b$-stimulated DCs.

DHEA synthetic derivate, HE2000, also proved to be beneficial in human infection. A pilot study showed a $50 \%$ reduction in Plasmodium falciparum blood levels in 41 out of 42 patients enrolled, thereafter achieving undetectable parasitemia levels in $76 \%$ of them [77]. A randomized, 
double-blind, placebo-controlled study showed that HE2000 administration induced significantly sustained decreases in IL- $1 \beta$, TNF- $\alpha$, IL- 6 , and Cox- 2 transcripts and although $\mathrm{CD} 4+\mathrm{T}$ cell numbers remain unaltered, patients demonstrated a significant decrease in viral load and a significant increase in CD8+ T cell responses after HE2000 administration [27]. Noteworthy, as shown by another study, HE2000 lowered by $42.2 \%$ the incidence of tuberculosis coinfection in AIDS patients while also reduced the cumulative incidence of opportunistic infections [78]. Unfortunately, despite the positive results obtained in phase I and II studies, currently there are no ongoing clinical trials exploring the effects of HE2000. Finally, at present we are studying the modulatory features of 7-oxo-DHEA, a DHEA natural derivative, on the immune response against TB in the context of HIV infection.

\section{Concluding Remarks}

Immune endocrine interactions during infectious diseases may determine the failure or success of the immune response. This is particularly true for chronic infections like HIV or $\mathrm{TB}$, in which pathogens and immune system coexist in a long struggle. Here we show the importance of DHEA and its derivatives on immunity to intracellular pathogens like HIV and $M t b$ through its action on Th1 and CD8 responses. These compounds also exert anti-inflammatory actions, which may be beneficial in the context of chronic infections, especially during HIV-TB coinfection.

This dual function of DHEA makes it very suitable as an immune-therapy for the prevention and treatment of TB in the context of HIV infection.

\section{Conflict of Interests}

The authors declare that there is no conflict of interests regarding the publication of this paper.

\section{Acknowledgments}

This work was supported by Agencia Nacional de Promoción Científica y Tecnológica (PICT 2010-0656 and PICT 20120059 to Maria Florencia Quiroga) and the University of Buenos Aires (UBACyT 20020120200050BA to Maria Florencia Quiroga). Guadalupe Veronica Suarez is a Postgraduate Fellow of ANPCyT; Maria Belen Vecchione and Matias Tomas Angerami are Postgraduate Fellows of CONICET. Maria Florencia Quiroga and Oscar Adelmo Bottasso are members of the Research Career at CONICET. The authors thank Dr. Gabriela Turk, Dr. Horacio Salomon, and Dr. Natalia Laufer for continuous support, Sergio Mazzini for thoroughly revising the paper, and Dr. Graciela Ben and Dr. Héctor Pérez for assistance in recruiting the patients in HIVTB studies.

\section{References}

[1] H. Getahun, C. Gunneberg, R. Granich, and P. Nunn, "HIV infection-associated tuberculosis: the epidemiology and the response," Clinical Infectious Diseases, vol. 50, supplement 3, pp. S201-S207, 2010.

[2] E. L. Corbett, C. J. Watt, N. Walker et al., "The growing burden of tuberculosis: global trends and interactions with the HIV epidemic," Archives of Internal Medicine, vol. 163, no. 9, pp. 1009-1021, 2003.

[3] P. A. Selwyn, D. Hartel, V. A. Lewis et al., "A prospective study of the risk of tuberculosis among intravenous drug users with human immunodeficiency virus infection," The New England Journal of Medicine, vol. 320, no. 9, pp. 545-550, 1989.

[4] C. Whalen, C. R. Horsburgh, D. Hom, C. Lahart, M. Simberkoff, and J. Ellner, "Accelerated course of human immunodeficiency virus infection after tuberculosis," The American Journal of Respiratory and Critical Care Medicine, vol. 151, no. 1, pp. 129135, 1995.

[5] K. Modjarrad and S. H. Vermund, "Effect of treating coinfections on HIV-1 viral load: a systematic review," The Lancet Infectious Diseases, vol. 10, no. 7, pp. 455-463, 2010.

[6] J. E. Gomez and J. D. McKinney, "M. tuberculosis persistence, latency, and drug tolerance," Tuberculosis, vol. 84, no. 1-2, pp. 29-44, 2004.

[7] C. E. Barry Jr., H. I. Boshoff, V. Dartois et al., "The spectrum of latent tuberculosis: rethinking the biology and intervention strategies," Nature Reviews Microbiology, vol. 7, no. 12, pp. 845855, 2009.

[8] J. D. Ernst, "The immunological life cycle of tuberculosis," Nature Reviews Immunology, vol. 12, no. 8, pp. 581-591, 2012.

[9] N. Salahuddin, F. Ali, Z. Hasan, N. Rao, M. Aqeel, and F. Mahmood, "Vitamin D accelerates clinical recovery from tuberculosis: results of the SUCCINCT Study [Supplementary Cholecalciferol in recovery from tuberculosis]. A randomized, placebo-controlled, clinical trial of vitamin D supplementation in patients with pulmonary tuberculosis"' BMC Infectious Diseases, vol. 13, article 22, 2013.

[10] J. L. Johnson, E. Ssekasanvu, A. Okwera et al., "Randomized trial of adjunctive interleukin-2 in adults with pulmonary tuberculosis," The American Journal of Respiratory and Critical Care Medicine, vol. 168, no. 2, pp. 185-191, 2003.

[11] R. Suarez-Mendez, I. Garcia-Garcia, N. Fernandez-Olivera et al., "Adjuvant interferon gamma in patients with drugresistant pulmonary tuberculosis: a pilot study," BMC Infectious Diseases, vol. 4, article 44, 2004.

[12] R. S. Wallis, P. Kyambadde, J. L. Johnson et al., "A study of the safety, immunology, virology, and microbiology of adjunctive etanercept in HIV-1-associated tuberculosis," AIDS, vol. 18, no. 2, pp. 257-264, 2004.

[13] K. Dheda, S. K. Schwander, B. Zhu, R. N. Van Zyl-Smit, and Y. Zhang, "The immunology of tuberculosis: from bench to bedside," Respirology, vol. 15, no. 3, pp. 433-450, 2010.

[14] A. del Rey, H. Furukawa, G. Monge-Arditi, A. Kabiersch, K.H. Voigt, and H. O. Besedovsky, "Alterations in the pituitaryadrenal axis of adult mice following neonatal exposure to interleukin-1," Brain, Behavior, and Immunity, vol. 10, no. 3, pp. 235-248, 1996.

[15] G. P. Chrousos, "The hypothalamic-pituitary-adrenal axis and immune-mediated inflammation," The New England Journal of Medicine, vol. 332, no. 20, pp. 1351-1362, 1995.

[16] I. J. Elenkov, R. L. Wilder, G. P. Chrousos, and E. S. Vizi, "The sympathetic nerve-an integrative interface between two supersystems: the brain and the immune system," Pharmacological Reviews, vol. 52, no. 4, pp. 595-638, 2000. 
[17] F. Eskandari and E. M. Sternberg, "Neural-immune interactions in health and disease," Annals of the New York Academy of Sciences, vol. 966, pp. 20-27, 2002.

[18] A. R. Pérez, O. Bottasso, and W. Savino, "The impact of infectious diseases upon neuroendocrine circuits," NeuroImmunoModulation, vol. 16, no. 2, pp. 96-105, 2009.

[19] E. Roggero, A. R. Pérez, M. Tamae-Kakazu et al., "Endogenous glucocorticoids cause thymus atrophy but are protective during acute Trypanosoma cruzi infection," The Journal of Endocrinology, vol. 190, no. 2, pp. 495-503, 2006.

[20] J. M. Busillo and J. A. Cidlowski, "The five Rs of glucocorticoid action during inflammation: ready, reinforce, repress, resolve, and restore," Trends in Endocrinology and Metabolism, vol. 24, no. 3, pp. 109-119, 2013.

[21] K. L. Blauer, M. Poth, W. M. Rogers, and E. W. Bernton, "Dehydroepiandrosterone antagonizes the suppressive effects of dexamethasone on lymphocyte proliferation," Endocrinology, vol. 129, no. 6, pp. 3174-3179, 1991.

[22] J. D. Ashwell, F. W. M. Lu, and M. S. Vacchio, "Glucocorticoids in T cell development and function," Annual Review of Immunology, vol. 18, pp. 309-345, 2000.

[23] A. Kasperska-Zajac, "Asthma and dehydroepiandrosterone (DHEA): facts and hypotheses," Inflammation, vol. 33, no. 5, pp. 320-324, 2010.

[24] V. Brazão, F. H. Santello, L. C. Caetano, M. Del Vecchio Filipin, M. Paula Alonso Toldo, and J. C. do Prado, "Immunomodulatory effects of zinc and DHEA on the Th-1 immune response in rats infected with Trypanosoma cruzi," Immunobiology, vol. 215, no. 5, pp. 427-434, 2010.

[25] R. Hernández-Pando, D. Aguilar-Leon, H. Orozco et al., "16 $\alpha$ Bromoepiandrosterone restores $\mathrm{T}$ helper cell type 1 activity and accelerates chemotherapy-induced bacterial clearance in a model of progressive pulmonary tuberculosis," The Journal of Infectious Diseases, vol. 191, no. 2, pp. 299-306, 2005.

[26] R. M. Loria, D. H. Conrad, T. Huff, H. Carter, and D. Ben-Nathan, "Androstenetriol and androstenediol. Protection against lethal radiation and restoration of immunity after radiation injury," Annals of the New York Academy of Sciences, vol. 917, pp. 860-867, 2000.

[27] C. Reading, C. Dowding, B. Schramm et al., "Improvement in immune parameters and human immunodeficiency virus-1 viral response in individuals treated with $16 \alpha$-bromoepiandrosterone (HE2000)," Clinical Microbiology and Infection, vol. 12, no. 11, pp. 1082-1088, 2006.

[28] F. Kelestimur, Y. Unlu, M. Ozesmi, and I. Tolu, "A hormonal and radiological evaluation of adrenal gland in patients with acute or chronic pulmonary tuberculosis," Clinical Endocrinology, vol. 41, no. 1, pp. 53-56, 1994.

[29] G. P. Chrousos and E. D. Zapanti, "Hypothalamic-pituitaryadrenal axis in HIV infection and disease," Endocrinology and Metabolism Clinics of North America, vol. 43, pp. 791-806, 2014.

[30] K. Keven, A. R. Uysal, and G. Erdogan, "Adrenal function during tuberculous infection and effects of antituberculosis treatment on endogenous and exogenous steroids," International Journal of Tuberculosis and Lung Disease, vol. 2, no. 5, pp. 419-424, 1998.

[31] O. Bottasso, M. L. Bay, H. Besedovsky, and A. del Rey, "Adverse neuro-immune-endocrine interactions in patients with active tuberculosis," Molecular and Cellular Neuroscience, vol. 53, pp. 77-85, 2013.
[32] R. J. North and Y.-J. Jung, "Immunity to tuberculosis," Annual Review of Immunology, vol. 22, pp. 599-623, 2004.

[33] J. Chan and J. Flynn, "The immunological aspects of latency in tuberculosis," Clinical Immunology, vol. 110, no. 1, pp. 2-12, 2004.

[34] A. A. Chackerian, J. M. Alt, T. V. Perera, C. C. Dascher, and S. M. Behar, "Dissemination of Mycobacterium tuberculosis is influenced by host factors and precedes the initiation of T-cell immunity, Infection and Immunity, vol. 70, no. 8, pp. 45014509, 2002.

[35] J. L. Flynn, M. M. Goldstein, K. J. Triebold, B. Koller, and B. R. Bloom, "Major histocompatibility complex class I-restricted T cells are required for resistance to Mycobacterium tuberculosis infection," Proceedings of the National Academy of Sciences of the United States of America, vol. 89, no. 24, pp. 12013-12017, 1992.

[36] C. G. Feng, A. G. D. Bean, H. Hooi, H. Briscoe, and W. J. Britton, "Increase in gamma interferon-secreting $\mathrm{CD}^{+}$, as well as $\mathrm{CD}^{+}, \mathrm{T}$ cells in lungs following aerosol infection with Mycobacterium tuberculosis," Infection and Immunity, vol. 67, no. 7, pp. 3242-3247, 1999.

[37] C. H. Ladel, C. Blum, A. Dreher, K. Reifenberg, and S. H. E. Kaufmann, "Protective role of $\gamma / \delta$ T cells and $\alpha / \beta$ T cells in tuberculosis," European Journal of Immunology, vol. 25, no. 10, pp. 2877-2881, 1995.

[38] H. Bruns, C. Meinken, P. Schauenberg et al., "Anti-TNF immunotherapy reduces CD8+ T cell-mediated antimicrobial activity against Mycobacterium tuberculosis in humans," The Journal of Clinical Investigation, vol. 119, no. 5, pp. 1167-1177, 2009.

[39] G. J. Fox, N. V. Nhung, D. N. Sy et al., "Contact investigation in households of patients with tuberculosis in Hanoi, Vietnam: a prospective cohort study," PLOS ONE, vol. 7, no. 11, Article ID e49880, 2012.

[40] A. D. Rey, C. V. Mahuad, V. V. Bozza et al., "Endocrine and cytokine responses in humans with pulmonary tuberculosis," Brain, Behavior, and Immunity, vol. 21, no. 2, pp. 171-179, 2007.

[41] E. L. York, D. A. Enarson, E. J. Nobert, F. A. Fanning, and B. J. Sproule, "Adrenocortical function in patients investigated for active tuberculosis," Chest, vol. 101, no. 5, pp. 1338-1341, 1992.

[42] F. Keleştimur, Z. Göktaş, I. Gülmez et al., "Low dose (1 $\mu \mathrm{g})$ adrenocorticotropin stimulation test in the evaluation of hypothalamo-pituitary-adrenal axis in patients with active pulmonary tuberculosis," Journal of Endocrinological Investigation, vol. 23, no. 4, pp. 235-239, 2000.

[43] I. M. Orme, A. D. Roberts, J. P. Griffin, and J. S. Abrams, "Cytokine secretion by CD4 T lymphocytes acquired in response to Mycobacterium tuberculosisinfection," Journal of Immunology, vol. 151, no. 1, pp. 518-525, 1993.

[44] V. Bozza, L. D’Attilio, G. Didoli et al., "The adrenal steroid response during tuberculosis and its effects on the mycobacterial-driven IFN-gamma production of patients and their household contacts," Annals of the New York Academy of Sciences, vol. 1153, pp. 247-255, 2009.

[45] L. D’Attilio, V. V. Bozza, N. Santucci et al., “TGF- $\beta$ neutralization abrogates the inhibited DHEA production mediated by factors released from M. tuberculosis-stimulated PBMC," Annals of the New York Academy of Sciences, vol. 1262, no. 1, pp. $1-9,2012$.

[46] S. Moir, T.-W. Chun, and A. S. Fauci, "Pathogenic mechanisms of HIV disease," Annual Review of Pathology, vol. 6, pp. 223-248, 2011. 
[47] S. M. Kahan, E. J. Wherry, and A. J. Zajac, "T cell exhaustion during persistent viral infections," Virology, vol. 479-480, pp. 180-193, 2015.

[48] A. R. Hersperger, F. Pereyra, M. Nason et al., "Perforin expression directly ex vivo by HIV-specific $\mathrm{CD}^{+} \mathrm{T}$-cells is a correlate of HIV elite control," PLoS Pathogens, vol. 6, no. 5, Article ID e1000917, 2010.

[49] S. A. Migueles, C. M. Osborne, C. Royce et al., "Lytic granule loading of $\mathrm{CD}^{+} \mathrm{T}$ cells is required for HIV-infected cell elimination associated with immune control," Immunity, vol. 29, no. 6, pp. 1009-1021, 2008.

[50] T. Miura, M. A. Brockman, A. Schneidewind et al., "HLAB57/B 5801 human immunodeficiency virus type 1 elite controllers select for rare gag variants associated with reduced viral replication capacity and strong cytotoxic T-lymphotye recognition," Journal of Virology, vol. 83, no. 6, pp. 2743-2755, 2009.

[51] M. Clerici, M. L. Fusi, S. Ruzzante et al., "Type 1 and type 2 cytokines in HIV infection-a possible role in apoptosis and disease progression," Annals of Medicine, vol. 29, no. 3, pp. 185$188,1997$.

[52] M. A. Reuter, C. Pombo, and M. R. Betts, "Cytokine production and dysregulation in HIV pathogenesis: lessons for development of therapeutics and vaccines," Cytokine \& Growth Factor Reviews, vol. 23, no. 4-5, pp. 181-191, 2012.

[53] L.-L. Liu, Y. Qin, J.-F. Cai et al., "Th17/Treg imbalance in adult patients with minimal change nephrotic syndrome," Clinical Immunology, vol. 139, no. 3, pp. 314-320, 2011.

[54] B. Kanwar, D. Favre, and J. M. McCune, "Th17 and regulatory T cells: implications for AIDS pathogenesis," Current Opinion in HIV and AIDS, vol. 5, no. 2, pp. 151-157, 2010.

[55] J. Mayo, J. Collazos, E. Martinez, and S. Ibarra, "Adrenal function in the human immunodeficiency virus-infected patient," Archives of Internal Medicine, vol. 162, no. 10, pp. 1095-1098, 2002.

[56] S. T. Azar and J. C. Melby, "Hypothalamic-pituitary-adrenal function in non-AIDS patients with advanced HIV infection," The American Journal of the Medical Sciences, vol. 305, no. 5, pp. 321-325, 1993.

[57] N. Christeff, N. Gherbi, O. Mammes et al., "Serum cortisol and DHEA concentrations during HIV infection," Psychoneuroendocrinology, vol. 22, supplement 1, pp. S11-S18, 1997.

[58] A. Costa, R. E. Nappi, F. Polatti, A. Poma, A. B. Grossman, and G. Nappi, "Stimulating effect of HIV-1 coat protein gp120 on corticotropin-releasing hormone and arginine vasopressin in the rat hypothalamus: involvement of nitric oxide," Experimental Neurology, vol. 166, no. 2, pp. 376-384, 2000.

[59] T. Kino and G. P. Chrousos, "Glucocorticoid and mineralocorticoid resistance/hypersensitivity syndromes," The Journal of Endocrinology, vol. 169, no. 3, pp. 437-445, 2001.

[60] T. Kino and G. P. Chrousos, "Human immunodeficiency virus type-1 accessory protein Vpr: a causative agent of the AIDSrelated insulin resistance/lipodystrophy syndrome?" Annals of the New York Academy of Sciences, vol. 1024, pp. 153-167, 2004.

[61] T. L. Wisniewski, C. W. Hilton, E. V. Morse, and F. Svec, "The relationship of serum DHEA-S and cortisol levels to measures of immune function in human immunodeficiency virus-related illness," American Journal of the Medical Sciences, vol. 305, no. 2, pp. 79-83, 1993.
[62] M. Clerici, D. Trabattoni, S. Piconi et al., "A possible role for the cortisol/anticortisols imbalance in the progression of human immunodeficiency virus," Psychoneuroendocrinology, vol. 22, supplement 1, pp. S27-S31, 1997.

[63] M. N. Silverman, B. D. Pearce, C. A. Biron, and A. H. Miller, "Immune modulation of the hypothalamic-pituitary-adrenal (HPA) axis during viral infection," Viral Immunology, vol. 18, no. 1, pp. 41-78, 2005.

[64] G. Norbiato, M. Bevilacqua, T. Vago, and M. Clerici, "Glucocorticoid resistance and the immune function in the immunodeficiency syndrome," Annals of the New York Academy of Sciences, vol. 840, pp. 835-847, 1998.

[65] M. F. Quiroga, M. T. Angerami, N. Santucci et al., "Dynamics of adrenal steroids are related to variations in Th1 and Treg populations during mycobacterium tuberculosis infection in HIV positive persons," PLoS ONE, vol. 7, no. 3, Article ID e33061, 2012.

[66] D. A. Horwitz, "Identity of mysterious CD $4^{+} \mathrm{CD} 25^{-} \mathrm{Foxp}^{3+}$ cells in SLE," Arthritis Research \& Therapy, vol. 12, no. 1, p. 101, 2010.

[67] H.-X. Yang, W. Zhang, L.-D. Zhao et al., "Are $\mathrm{CD} 4^{+} \mathrm{CD} 25^{-}$Foxp $^{+}$cells in untreated new-onset lupus patients regulatory T cells?" Arthritis Research and Therapy, vol. 11, no. 5, article R153, 2009.

[68] M. Bonelli, A. Savitskaya, C.-W. Steiner, E. Rath, J. S. Smolen, and C. Scheinecker, "Phenotypic and functional analysis of $\mathrm{CD} 4^{+} \mathrm{CD} 25^{-}$Foxp $^{+} \mathrm{T}$ cells in patients with systemic lupus erythematosus," Journal of Immunology, vol. 182, no. 3, pp. 16891695, 2009.

[69] S. M. Kaech and W. Cui, "Transcriptional control of effector and memory $\mathrm{CD}^{+} \mathrm{T}$ cell differentiation," Nature Reviews Immunology, vol. 12, no. 11, pp. 749-761, 2012.

[70] L. E. Kihel, "Oxidative metabolism of dehydroepiandrosterone (DHEA) and biologically active oxygenated metabolites of DHEA and epiandrosterone (EpiA) - recent reports," Steroids, vol. 77, no. 1-2, pp. 10-26, 2012.

[71] M. Schmidt, M. Kreutz, G. Löffler, J. Schölmerich, and R. H. Sträub, "Conversion of dehydroepiandrosterone to downstream steroid hormones in macrophages," The Journal of Endocrinology, vol. 164, no. 2, pp. 161-169, 2000.

[72] D. Ben-Nathan, D. A. Padgett, and R. M. Loria, "Androstenediol and dehydroepiandrosterone protect mice against lethal bacterial infections and lipopolysaccharide toxicity," Journal of Medical Microbiology, vol. 48, no. 5, pp. 425-431, 1999.

[73] V. Brazão, F. H. Santello, L. C. Caetano, M. Del Vecchio Filipin, M. Paula Alonso Toldo, and J. C. do Prado, "Immunomodulatory effects of zinc and DHEA on the Th-1 immune response in rats infected with Trypanosoma cruzi," Immunobiology, vol. 215, no. 5, pp. 427-434, 2010.

[74] D. A. Padgett and R. M. Loria, "In vitro potentiation of lymphocyte activation by dehydroepiandrosterone, androstenediol, and androstenetriol," Journal of Immunology, vol. 153, no. 4, pp. 1544-1552, 1994.

[75] Y. Sakakura, Y. Nakagawa, and T. Ohzeki, "Differential effect of DHEA on mitogen-induced proliferation of T and B lymphocytes," The Journal of Steroid Biochemistry and Molecular Biology, vol. 99, no. 2-3, pp. 115-120, 2006.

[76] M. Angerami, G. Suarez, M. F. Pascutti, H. Salomon, O. Bottasso, and M. F. Quiroga, "Modulation of the phenotype and function of Mycobacterium tuberculosis-stimulated dendritic cells by adrenal steroids," International Immunology, vol. 25, no. 7, pp. 405-411, 2013. 
[77] J. M. Frincke, D. R. Stickney, N. Onizuka-Handa et al., "Reduction of parasite levels in patients with uncomplicated malaria by treatment with HE2000," The American Journal of Tropical Medicine and Hygiene, vol. 76, no. 2, pp. 232-236, 2007.

[78] D. R. Stickney, Z. Noveljic, A. Garsd, D. A. Destiche, and J. M. Frincke, "Safety and activity of the immune modulator HE2000 on the incidence of tuberculosis and other opportunistic infections in AIDS patients," Antimicrobial Agents and Chemotherapy, vol. 51, no. 7, pp. 2639-2641, 2007. 

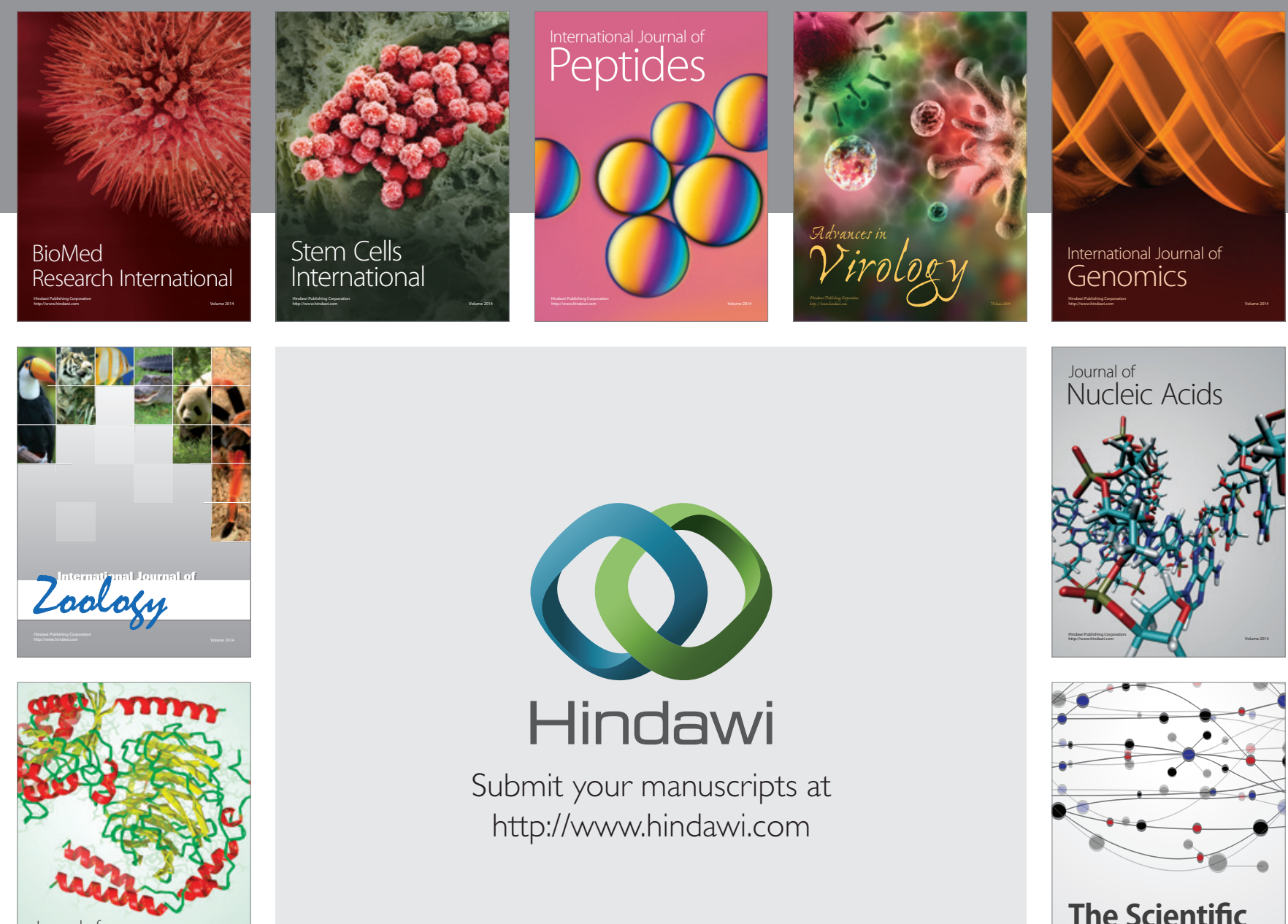

Submit your manuscripts at

http://www.hindawi.com

Journal of
Signal Transduction
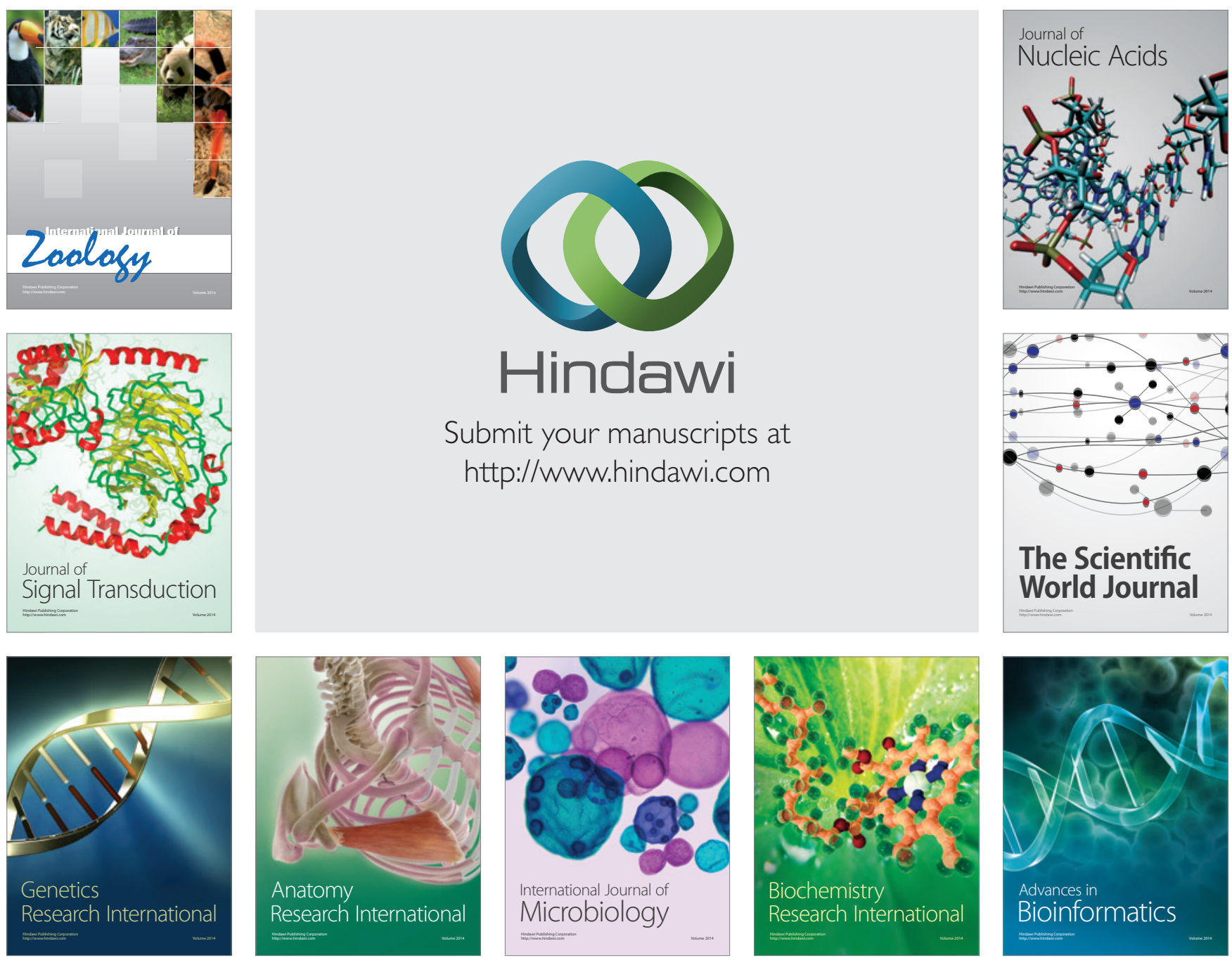

The Scientific World Journal
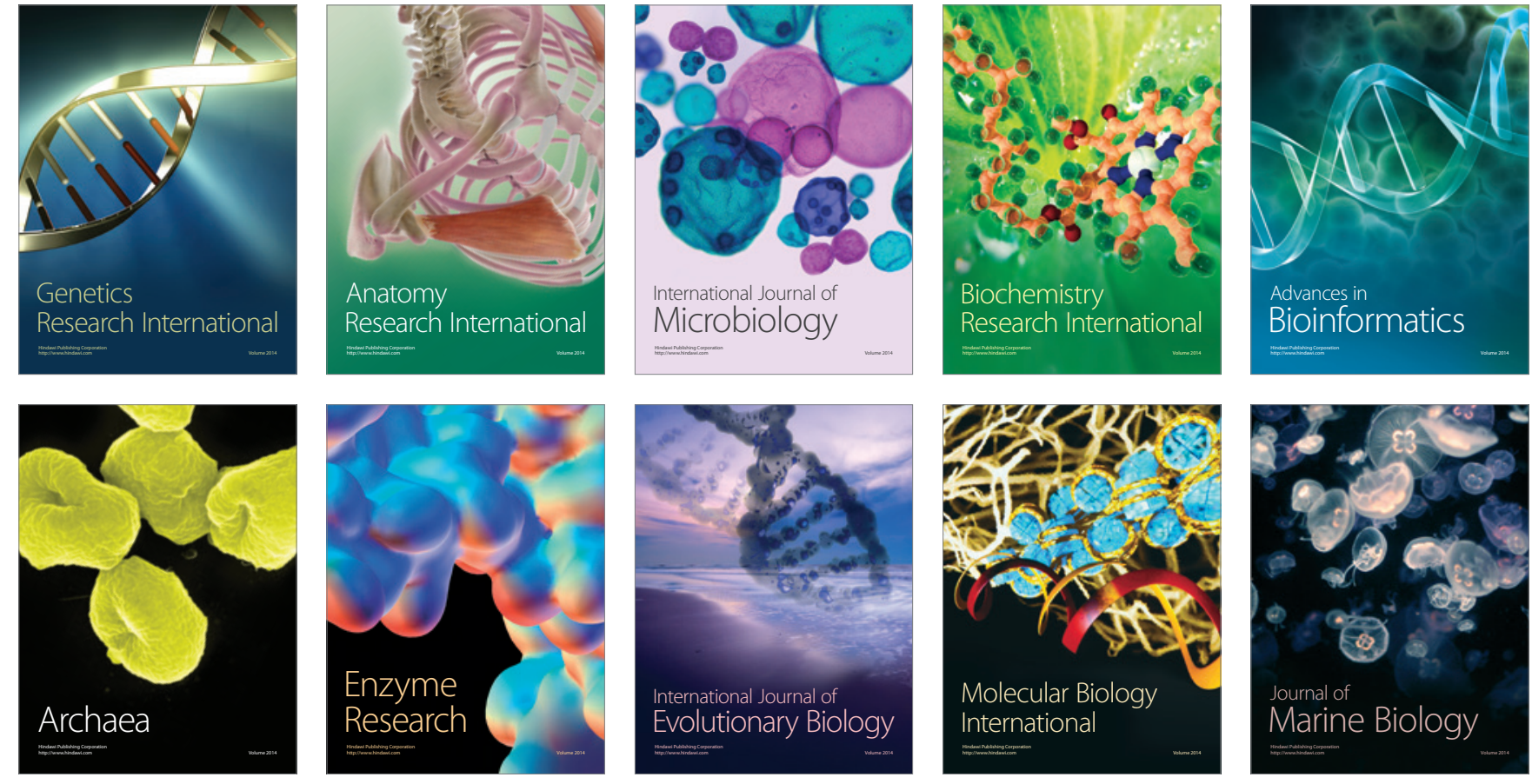